\title{
Balmer continuum enhancement detected in a mini flare observed with IRIS
}

\author{
Reetika Joshi ${ }^{1,2} \odot$, Brigitte Schmieder ${ }^{1,3}$, Petr Heinzel ${ }^{4}$, James Tomin ${ }^{1}$, Ramesh Chandra ${ }^{2} \odot$, and Nicole Vilmer ${ }^{1}(\mathbb{0}$ \\ ${ }^{1}$ LESIA, Observatoire de Paris, Université PSL, CNRS, Sorbonne Université, Université de Paris, 5 place Jules Janssen, \\ 92190 Meudon, France \\ e-mail: reetikajoshi.ntl@gmail.com \\ 2 Department of Physics, DSB Campus, Kumaun University, Nainital 263 001, India \\ 3 Centre for mathematical Plasma Astrophysics, Dept. of Mathematics, KU Leuven, 3001 Leuven, Belgium \\ 4 Astronomical Institute of the Czech Academy of Sciences, Fričova 298, 25165 Ondřejov, Czech Republic
}

Received 24 April 2021 / Accepted 23 July 2021

\begin{abstract}
Context. Optical and near-UV continuum emissions in flares contribute substantially to the flare energy budget. Two mechanisms play an important role for continuum emission in flares: hydrogen recombination after sudden ionization at chromospheric layers, and transportation of the energy radiatively from the chromosphere to lower layers in the atmosphere, the so-called back-warming.

Aims. The aim of the paper is to distinguish between these two mechanisms for the excess of the Balmer continuum observed in a flare.

Methods. We combined the observations of the Balmer continuum obtained with the Interface Region Imaging Spectrograph (IRIS) (spectra and slit-jaw images (SJIs) $2832 \AA$ ) and hard X-ray (HXR) emission detected by the Fermi/Gamma Burst Monitor (GBM) during a mini flare. The calibrated Balmer continuum was compared to non-local thermodynamic equilibrium (LTE) radiative transfer flare models, and the radiated energy was estimated. Assuming thick target HXR emission, we calculated the energy of the nonthermal electrons detected by the Fermi/GBM and compared it to the radiated energy.

Results. The favorable argument of a relation between the Balmer continuum excess and the HXR emission is that there is a good time coincidence between them. In addition, the shape of the maximum brightness in the 2832 SJIs, which is mainly due to this Balmer continuum excess, is similar to that of the Fermi/GBM light curve. The electron-beam flux estimated from Fermi/GBM between $10^{9}$ and $10^{10} \mathrm{erg} \mathrm{s}^{-1} \mathrm{~cm}^{-2}$ is consistent with the beam flux required in non-LTE radiative transfer models to obtain the excess of Balmer continuum emission observed in this IRIS spectra.

Conclusions. The low-energy input by nonthermal electrons above $20 \mathrm{keV}$ is sufficient to produce the enhancement in the Balmer continuum emission. This could be explained by the topology of the reconnection site. The reconnection starts in a tiny bald-patch region, which is transformed dynamically into an X-point current sheet. The size of the interacting region would be below the spatial resolution of the instrument.
\end{abstract}

Key words. Sun: chromosphere - Sun: flares - Sun: transition region

\section{Introduction}

The heating of the lower solar atmosphere during solar flares is an interesting and still open area in solar physics because it deals with the energy distribution in flares. This is directly related to the radiation emitted during a solar flare. The heated atmosphere produces the enhancement of emission in many lines and continua. The origin of optical continuum in flares comes from two mechanisms: the hydrogen recombination as for continua (Paschen, Balmer) in the chromosphere, and $\mathrm{H}^{-}$emission in the photosphere. The optical continuum enhancement is a reliable signature of the so-called white-light flare (WLF, Fletcher et al. 2011). Enhancement of the Balmer continuum below $3646 \AA$ has been reported in ground-based observations of flares (Neidig 1989) and recently in the Interface Region Imaging Spectrograph (IRIS, De Pontieu et al. 2014) spectra during strong X-class WLFs (Heinzel \& Kleint 2014; Kleint et al. 2016, 2017). Electron beams are often invoked to explain the WLF heating in the low chromosphere and photosphere. The enhancement in the Balmer continuum emission is produced in higher levels in the chromosphere. Heinzel \& Kleint (2014) compared the enhancement in the Balmer continuum with the light curves from RHESSI and found a good spatial and temporal correlation, which was explained by electron-beam heating and ionizing of the chromosphere. Then the subsequent recombination leads to Balmer-continuum emission. The chromosphere might produce the Balmer continuum emission enhancement, while the photosphere in some cases might produce the whitelight emission, which is probably due to radiative back-warming (Machado et al. 1989). This issue was discussed earlier in Ding et al. (2003) and Heinzel \& Kleint (2014) and was quantitatively modeled in Kleint et al. (2016).

A quasi-continuous enhancement in flares can be interpreted in three different ways. (i) It may be the Balmer continuum emission, superposed over the background spectrum. (ii) The continuum is due to contribution from the photosphere either by direct beam bombardment (but this could take place only for strong beams, such as in strong flares) or by radiative backwarming. (iii) Finally, the enhancement might be affected by the emission of the broad wings of Mg II lines. Heinzel \& Kleint (2014) took advantage of the IRIS $2832 \AA$ band spectra, in which the Mg II wings were not present, to identify the real Balmer continuum excess at the site of an X-class flare. 

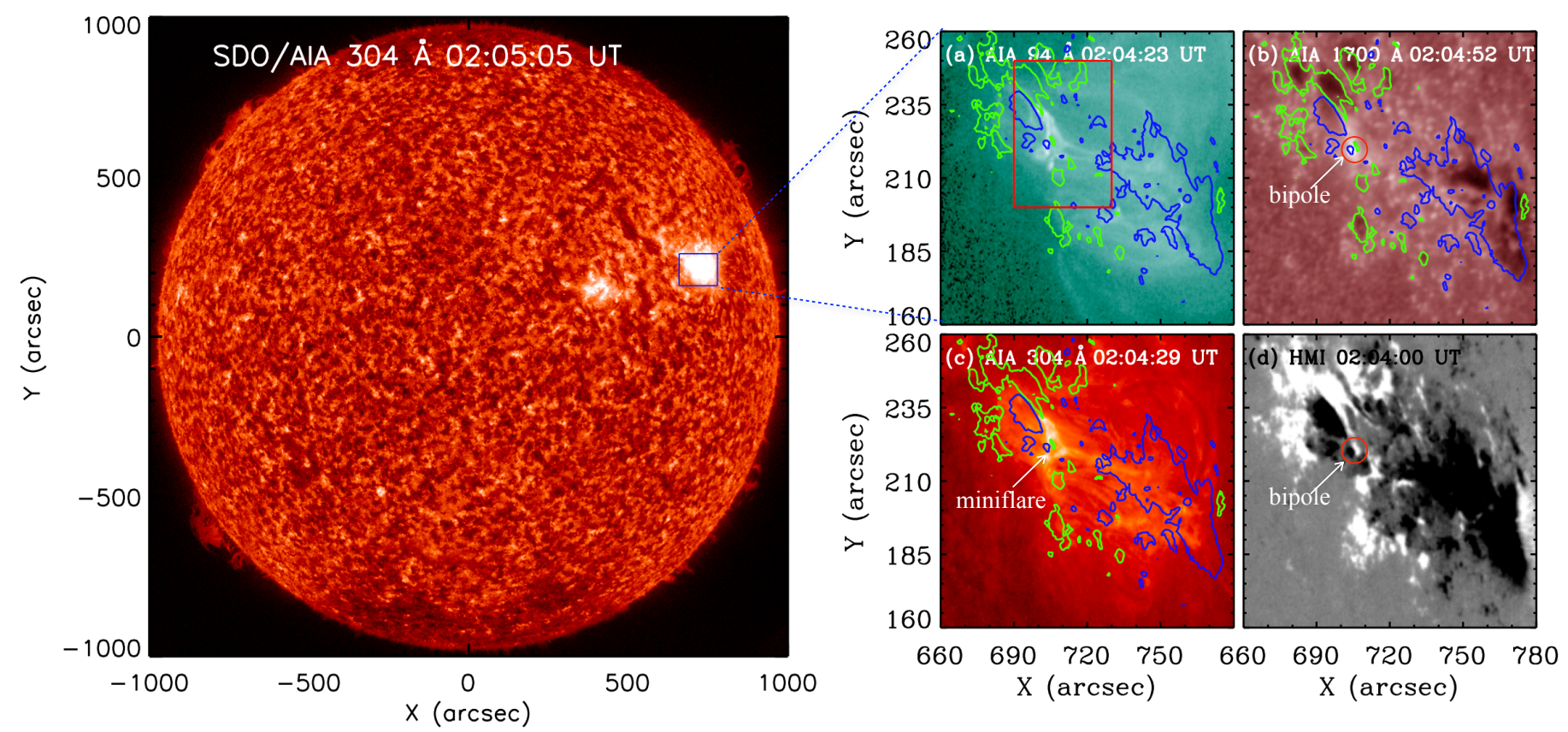

Fig. 1. Active region AR NOAA 12736 observed with AIA on March 22, 2019. Left panel: full disk in $304 \AA$ A. The blue box (field of view of the right panels) contains the only AR that is visible on the disk on that day. Right panels $(a, b, c)$ : AR in different AIA filters, in $94 \AA$, $1700 \AA$, and $304 \AA$ A superimposed with the contours of the magnetic field of strength \pm 300 Gauss. Panel $d$ : magnetic field observed with HMI. The bipole (small circle in panels $b$ and $d$ ) is responsible for the reconnection. The mini flare is indicated by an arrow in panel $c$. The box in panel $a$ is the field of view of Fig. 2.

Recently, a micro flare observed in multiple wavelengths by Atmospheric Imaging Assembly (AIA, Lemen et al. 2012) on board the Solar Dynamics Observatory (SDO, Pesnell et al. 2012) and IRIS puzzled us as the Balmer continuum also appeared to be enhanced, even in a weak flare. This micro flare, which we called mini flare in previous papers, occurred at the base of a solar jet on March 22, 2019. The analysis of the vector magnetic field revealed a flux rope in the vicinity of the jet, and the magnetic reconnection took place in a bald-patch region in which the magnetic field lines were tangential to the photosphere (Joshi et al. 2020).

The proper motions of the photospheric magnetic polarities suggested that the twist of the flux rope was transferred during reconnection to the jet, generating a twisted jet. A detailed spectroscopic analysis of the mini flare associated with this jet was carried out with IRIS spectra (Joshi et al. 2021), Using the cloud model technique applied to the Mg II line profiles, we identified explosive clouds with supersonic Alfvén velocities. A stratification thermal model of the atmosphere at the time of the reconnection was proposed. The Mg II spectra show extended wings like in IRIS bombs (Peter et al. 2014; Grubecka et al. 2016). Twist and bald-patch reconnection were confirmed with the analysis of the IRIS spectra (Si IV, C II, and Mg II) by Joshi et al. (2020).

In this paper we extend the IRIS data analysis of the mini flare studied in Joshi et al. $(2020,2021)$ and present the Balmer continuum around $2832 \AA$ observed with IRIS (Sect. 2). Section 3 presents the IRIS spectra and $2832 \AA$ slit-jaw images (SJIs) analysis. Furthermore, we analyzed the hard X-ray (HXR) emission detected by Fermi/Gamma Burst Monitor (GBM) (Sect. 4). We discuss the possible non-local thermodynamic equilibrium (LTE) radiative transfer models, proposed in Kleint et al. (2016), deriving the radiated energy from the measurements of the Balmer continuum. We compare the radiative energy to the energy contained in nonthermal electrons as derived from HXR measurements. In conclusion, we conjecture that the Balmer continuum enhancement is due to the energy electron deposit produced during the flare (Sect. 5). We suggest that the energy deposit, even with a relatively low value, is sufficient because the reconnection is located in the low layers of the atmosphere in a tiny bald-patch region, as the magnetic topology of the region revealed (Joshi et al. 2020).

\section{Cotemporal observations}

\subsection{AIA and IRIS SJI}

The mini flare (GOES B6.7) occurred in the active region (AR NOAA 12736) that was located at N09 W60 on March 22, 2019. It was the only AR in the whole solar disk on that day (Fig. 1 left panel). AIA on board SDO allows us to display the images of the mini flare in all the channels from UV (1600 and $1700 \AA)$ to EUV $(94 \AA, 131 \AA, 171 \AA, 193 \AA, 211 \AA$, and $304 \AA)$ covering a broad temperature range from $4500 \mathrm{~K}$ to $10^{6} \mathrm{~K}$ (Figs. $1 \mathrm{a}-\mathrm{c}$ ). The $1700 \AA$ filter contains hotter coronal lines that are in emission in strong flares, leading to an enhancement of flare brightenings in this filter (Simões et al. 2019). Our mini flare being a weak flare, we may guess with a high probability that the enhancement visible at $1700 \AA$ is due to heating of the plasma in the minimum temperature region around $4500 \mathrm{~K}$. The topological configuration of the AR was analyzed previously (Joshi et al. 2020), showing that the AR formed by successively emerging magnetic fluxes (EMFs) adjacent to each other. The inversion line between two opposite polarities belonging to two different EMFs was the site of strong shear and magnetic reconnection, and a small bipole was identified as the origin of the mini flare in HMI magnetograms (Fig. 1d). The mini flare is in the central part of a bright, more or less north-south semicircle overlying the inversion line between positive and negative polarities. 

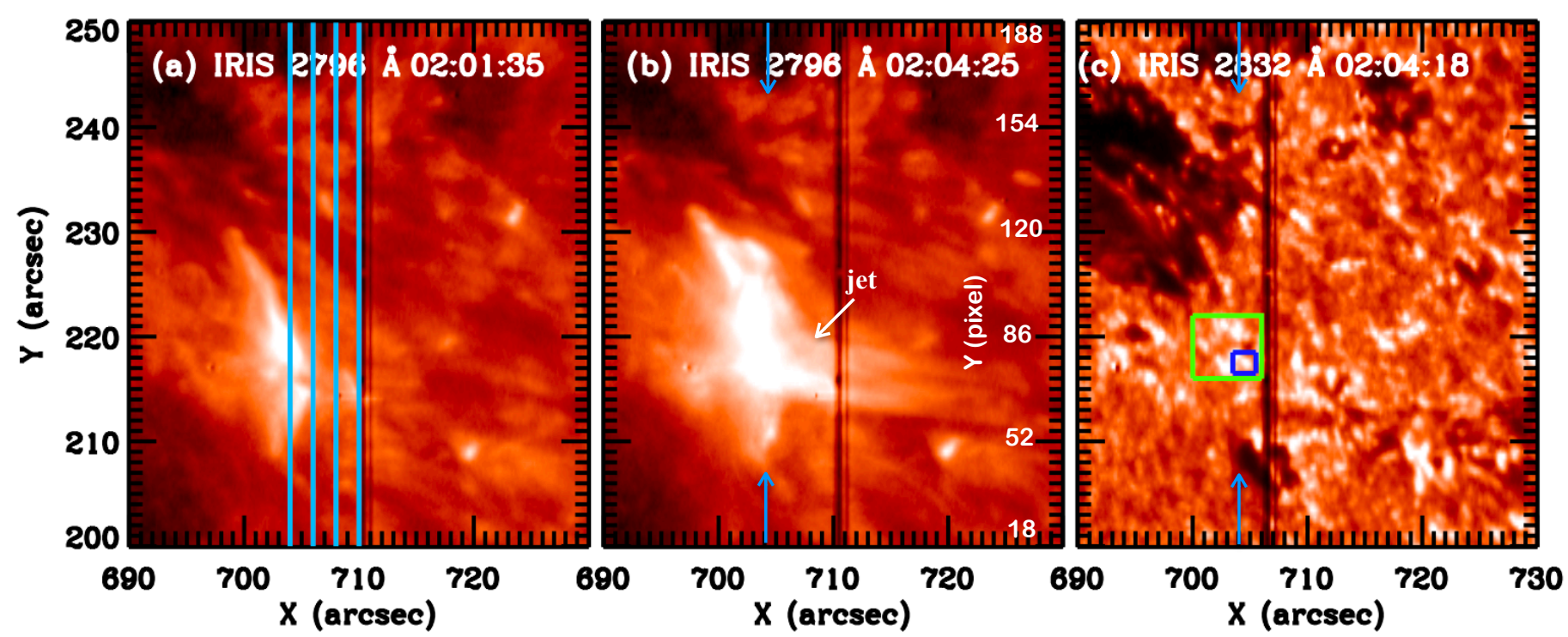

Fig. 2. IRIS SJI of the mini flare in $2796 \AA$ (panels $a, b$ ) and $2832 \AA$ (panel $c$ ) filters. Panel $a$ : the four vertical cyan lines show the four IRIS slit positions of the rasters. The vertical cyan arrows in panels $b, c$ indicate IRIS slit position 1, which crosses the mini flare (bipole) region presented in Fig. 1 (panels b-d). The green and blue boxes in panels $c$ are the fields of view used to compute the light curves of the bright point in Fig. 4.

IRIS contains three CCDs for the far-ultraviolet (FUV) and near-ultraviolet (NUV) spectra and one CCD for the SJIs. IRIS performed medium coarse rasters of four steps from 01:43:27 UT to 02:42:30 UT centered at $x=709^{\prime \prime}$ and $y=228^{\prime \prime}$ in the SJI field of view of $60^{\prime \prime} \times 68^{\prime \prime}$. The raster step size is $2^{\prime \prime}$, so that each spectral raster spans a field of view of $6^{\prime \prime} \times 62^{\prime \prime}$. The nominal spatial resolution is 0'.33. IRIS SJIs in Mg II 2896 filter and in $2832 \AA$ continuum filter are recorded with a $14 \mathrm{~s}$ cadence (see examples in Figs. $2 \mathrm{a}-\mathrm{c}$ ). The four slits are drawn in panel a. The first slit on the left crosses the mini flare. Slits 2, 3 , and 4 cross the jet on the right. In the 2832 continuum filter, small bright dots are observed at the location of the mini flare (green and blue boxes). For an accurate comparison between the AIA images and IRIS SJIs, we manually aligned these images by shifting the IRIS SJIs by $4^{\prime \prime}$ in $x$-axis and $3^{\prime \prime}$ in $y$-axis, as noted in the previous paper (Joshi et al. 2021). The relation between heliographic coordinates and pixels along the slit of the spectra is shown in Fig. $2 b$.

The IRIS Mg II spectra during the mini flare were analyzed in Joshi et al. (2021). Bidirectional flows $\left( \pm 200 \mathrm{~km} \mathrm{~s}^{-1}\right)$ were observed at the flare reconnection site, covering one or two pixels $y=79-80$ at 02:03:46 UT. At 02:04:28 UT, strong blueshifts in pixels $>80$ and redshifts a few minutes later were also observed, which we interpret as material of the jet going up and then falling back. The mini flare is definitely not a doubleribbon flare with many ribbons, as it is the case in Kleint et al. (2017). A bright continuum is detected all along the wavelength domain in the $\mathrm{Mg}$ II spectra at the flare site ( $y=70$ and 80 pixel) during a few minutes (Fig. 3). In this study we focus on the Balmer continuum far away from the extended $\mathrm{Mg}$ II line wings until $2835 \AA$ to be free of the emission of the $\mathrm{Mg}$ II line wings.

\subsection{Mini flare light curves}

We first checked the occurrence times of the soft X-ray (SXR) and HXR peaks with the bright-point intensity observed in the $2832 \AA$ filter by comparing the time variation of the X-ray flux with the intensity light curve of the $2832 \AA$ filter between 01:44 UT and 02:20 UT (Fig. 4). The SXR light curve of the flare is recorded by the GOES spacecraft in $0.5-4 \AA$ and $1-8 \AA$. The HXR count rates in different energy bands are recorded with the Gamma-ray Burst Monitor (GBM, Meegan et al. 2009) on board the Fermi spacecraft, which launched in 2008. Fermi/GBM fills the gap for HXR measurements for the solar physics community after the decommissioning of RHESSI. Fermi/GBM does not provide HXR images like RHESSI, therefore we cannot verify if the HXR emission comes from the flaring active region, but at that time, only one active region was present on the solar disk (Fig. 1 left panel), therefore the HXR emission is expected to come from the flaring active region. In the bottom panel of Fig. 4, Mg II $2832 \AA$ slit jaw intensity light curves are obtained by integrating the DN signal divided by the total number of pixels in the small boxes at the flare site (Fig. 2 green and blue boxes). We conclude that the occurrence times of the Balmer continuum enhancement, HXR emission, and the GOES light curves agree well for this flare.

\section{Balmer continuum enhancement}

As we described in the introduction, the enhancement of the Balmer continuum can be interpreted in different ways. To distinguish between the different mechanisms, we need to analyze the IRIS spectra in detail (Fig. 3) and calibrate the data to quantitatively compute the excess of the Balmer continuum emission.

\subsection{Intensity calibration spectra}

Using the IRIS radiometric calibration, we converted the data number (DN) into intensity units. We used the IDL routine iris_calib.pro and obtained the intensity value $\left(I_{\mathrm{c}}\right)$ in erg $\mathrm{cm}^{-2} \mathrm{~s}^{-1} \mathrm{sr}^{-1} \AA^{-1}$. The exposure time per slit position was $\approx 2 \mathrm{~s}$ for this conversion. The radiometric intensity calibration was made with a code developed by $\mathrm{H}$. Tian, with the following formula:

$I_{\mathrm{c}}=\frac{h c}{\lambda} \times n \times \frac{I_{\mathrm{o}}}{A_{\mathrm{eff}} \times d \times \omega}$.

The photon energy $h c / \lambda$ was calculated with Planck constant $h=$ $6.63 \times 10^{-27} \mathrm{erg} \mathrm{s}^{-1}$, and the speed of light $c=3 \times 10^{10} \mathrm{~cm} \mathrm{~s}^{-1}$. 

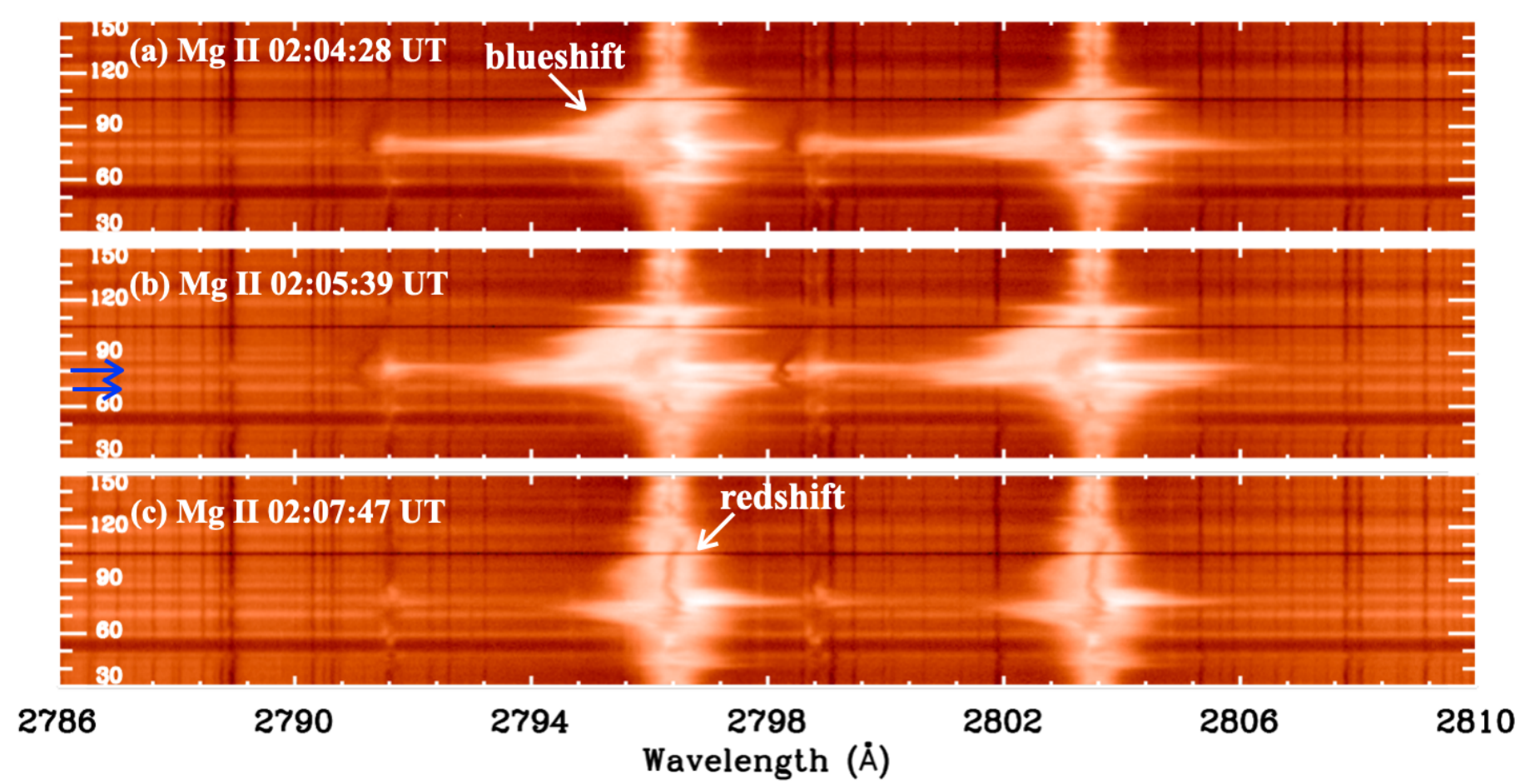

Fig. 3. Spectra of Mg II lines $\mathrm{h}$ and $\mathrm{k}$ in the Balmer continuum full wavelength range of IRIS (50 $\mathrm{A}$ ) during and after the mini flare times at the reconnection site along the first slit position (Figs. $2 \mathrm{a}-\mathrm{c}$ ). Bidirectional outflows are observed at the reconnection point $(y=80$ in panel a). Balmer continuum emission is visible in the three panels at two different pixels $(y=70,80)$ in wavelengths $10 \AA$ far from the $\mathrm{k}$ line core (see in panel $b$ the two horizontal blue arrows). Blueshifts and redshifts, indicated by two white arrows in the $\mathrm{Mg} \mathrm{II} \mathrm{k}$ line wings, correspond to the twisted expelled jet and material falling back (panels $a$ and $c$ ).

$n$ is the number of photons per $\mathrm{DN}$, it is 4 for FUV and 18 for NUV spectra. $I_{\mathrm{o}}$ is the observed intensity in $\mathrm{DN} \mathrm{s}^{-1}$ and $A_{\text {eff }}$ is the effective area in $\mathrm{cm}^{-2}$ and obtained through iris_get_response routine available in SolarSoftWare (SSW). The dispersion $d$ is taken in $\AA$ pixel $^{-1}$. The solid angle $\omega$ is calculated as $\omega=0.3327 \times 720 \times 0.33 \times 720 /\left(1.5 \times 10^{8}\right)^{2}$.

Figure 5 shows the relation between the DN signal in the range of $\mathrm{Mg}$ II $\mathrm{k}$ line and the calibrated data for the quiet Sun $(y=180$ pixel $)$. The $\mathrm{Mg}$ II k line peaks at $4.8 \times$ $10^{5} \mathrm{erg} \mathrm{s}^{-1} \mathrm{sr}^{-1} \mathrm{~cm}^{-2} \AA^{-1}$. At either end of the domain, the photospheric $\mathrm{Mg}$ II wing emission increases.

\subsection{IRIS spectra before the flare}

Figure 6 panel a shows the full range of the $\mathrm{Mg}$ II window spectra for the quiet Sun before the flare at 01:43:41 UT. This spectrum is our reference spectra. The full IRIS wavelength range focused on the $\mathrm{Mg}$ II lines and is extended from $2784 \AA$ to $2835 \AA$. This large domain allows us to analyze the Balmer continuum relatively far from the cores of the $\mathrm{Mg}$ II $\mathrm{h}$ and $\mathrm{k}$ lines. The spectral profiles of three pixels $(y=10,170,180)$ in the quiet Sun (Fig. 6b) can be compared to the global sun spectral profile shown in Pereira et al. (2013). This spectral profile has a U-shape with an increasing emission toward the two extremities of the spectral range, in the blue far wing of $\mathrm{k}$ and in the far red wing of $h$, both emissions corresponding to photosphere emissions (Figs. 6c and d, respectively). In the center of the profile lie the two chromospheric $\mathrm{h}$ and $\mathrm{k}$ lines cores in emission.

Even for the flare time, the spectral profile could be about the same as that detected in the IRIS spectrum outside the flare if there was no Balmer continuum enhancement. The fact that the "continuum" peaks in time with the HXR emission indicates a direct relation between the enhancement of the continuum and the nonthermal flux (Fig. 4). However, extended wings of Mg II lines during flares may also affect the continuum. Therefore we explored the wavelength range as far as possible from the $\mathrm{k}$ and $\mathrm{h}$ line cores at the flare time.

\subsection{IRIS spectra at the flare time}

The site of the flare is crossed by only one slit of the raster (slit 1), as was shown by Joshi et al. (2021). Figure 7 panel a shows the full wavelength range of the $\mathrm{Mg}$ II spectra along this slit at the time of the mini flare (02:04:28 UT). The reconnection site is detected by bidirectional outflows, principally blueshifts, which concerns one or two pixels along the slit. We note the U-shape profile with an increasing intensity at the two extremities of the wavelength range (Fig. 7b), similar to the spectral profile of the quiet Sun (Fig. 6b). It is difficult to detect the excess of the continuum emission without proceeding to a subtraction of the quiet-Sun profile. First we selected the pixels in which the Balmer continuum enhancement is more visible. To do this, we made cuts in the Mg II spectra (Fig. 7a) for different wavelengths in the continuum in the extreme left and right perpendicular to the dispersion direction (Fig. 8). Peaks in the continuum at the flare time are well visible for pixels 70 and 80 along the slit, which we selected for the following analysis.

By subtracting the background spectra in the whole IRIS Mg II wavelength band observed before the flare (01:43:41 UT) from the spectra during the flare (02:04:28 UT), we obtained the Balmer continuum excess. We verified that the signal is constant at the boundaries of the domain after the subtraction. Thus two different domains of the continuum were chosen, 2784-2791.5 $\AA$ and 2827-2834.5 $\AA$, to compute the 

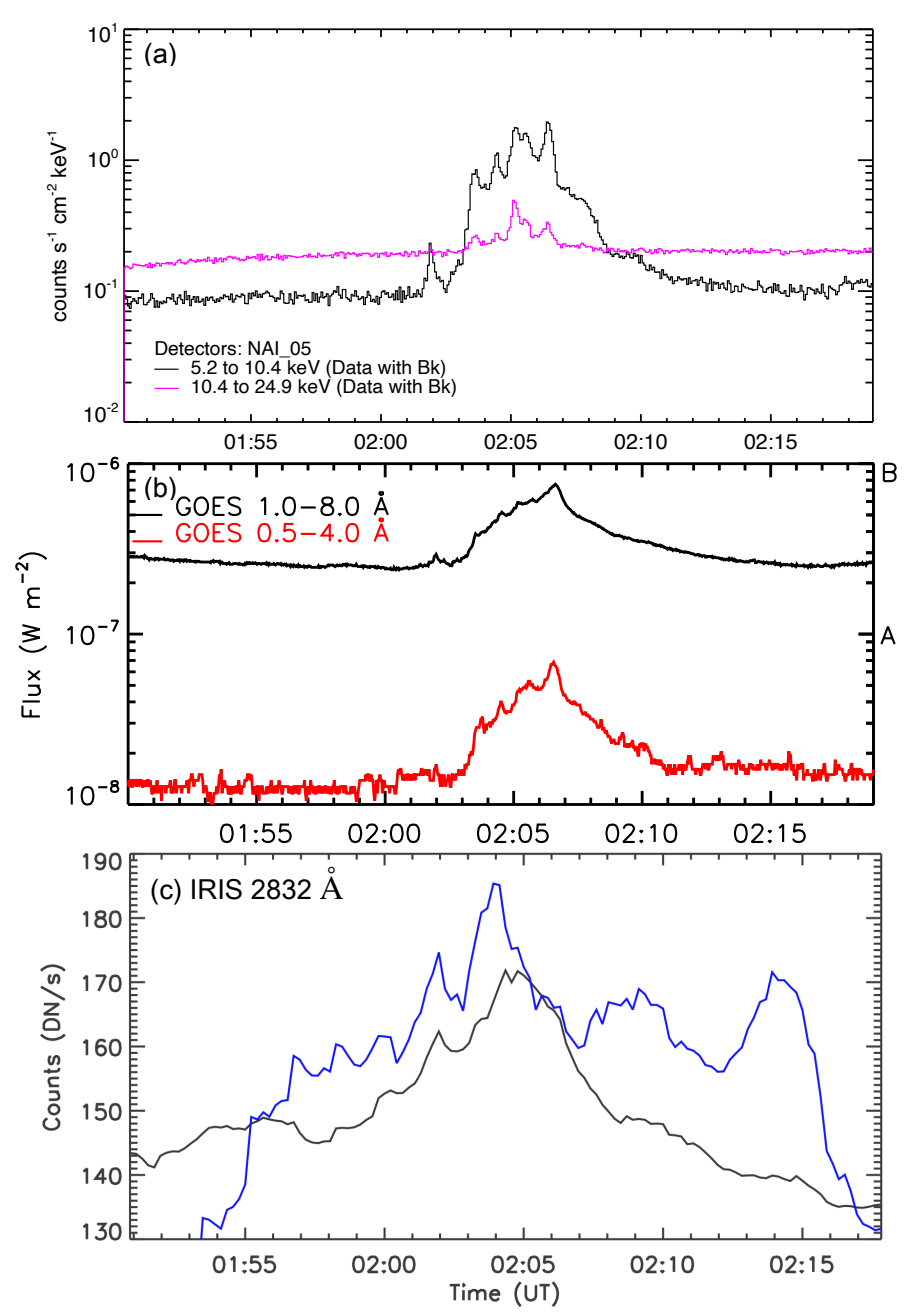

Fig. 4. Intensity variation at the flare site observed in Fermi/GBM, GOES, and IRIS SJIs. Panel a: X-ray count rates detected in two energy channels by Fermi/GBM. Panel $b$ : GOES light curve for the B6.7 class solar flare. It shows that the flare starts at 02:02 UT and peaks at $\approx 02: 06$ UT, with small peaks corresponding to GBM peaks. Panel $c$ : intensity light curves at the bright points in the IRIS $2832 \AA$ SJIs. The black and blue curves are the light curves over the green and blue boxes presented in Fig. 2d, respectively. The DN signal at the flare base is integrated and divided by the total number of pixels.

intensity variation (Figs. 7c, $\mathrm{d}$ for the flare time; e, $\mathrm{f}$ for the preflare time), and finally the subtraction of both intensities (Figs. 7g, h). The difference intensity plots show a constant continuum enhancement in each selected domain of about 1.5 to $1.75 \times 10^{5} \mathrm{erg} \mathrm{s}^{-1} \mathrm{sr}^{-1} \mathrm{~cm}^{-2} \AA^{-1}$. The superimposed residual signal is weak due to optically thin Balmer emission, but it is significant. The enhancement of the Balmer continuum corresponds to an increase of about $50 \%$ over the preflare level during the flare.

\subsection{IRIS SJI $2832 \AA$ filter}

The SJIs in the $2832 \AA$ filter show a brightening at the location of the flare around 02:05 UT (Fig. 2). In Fig. 4 we estimate the increase in brightening in the small box (Fig. 2c) by $50 \%$ $((180-120) / 120)$ between the time of the preflare $(01: 44$ UT) and the flare time (02:04 UT). The SJI $2832 \AA$ filter includes the two Mg II k and h lines and a large wavelength domain of the continuum. The 2832 filter transmission profile $f(\lambda)$ has two peaks: one peak around $2830 \AA$, and the second peak close to

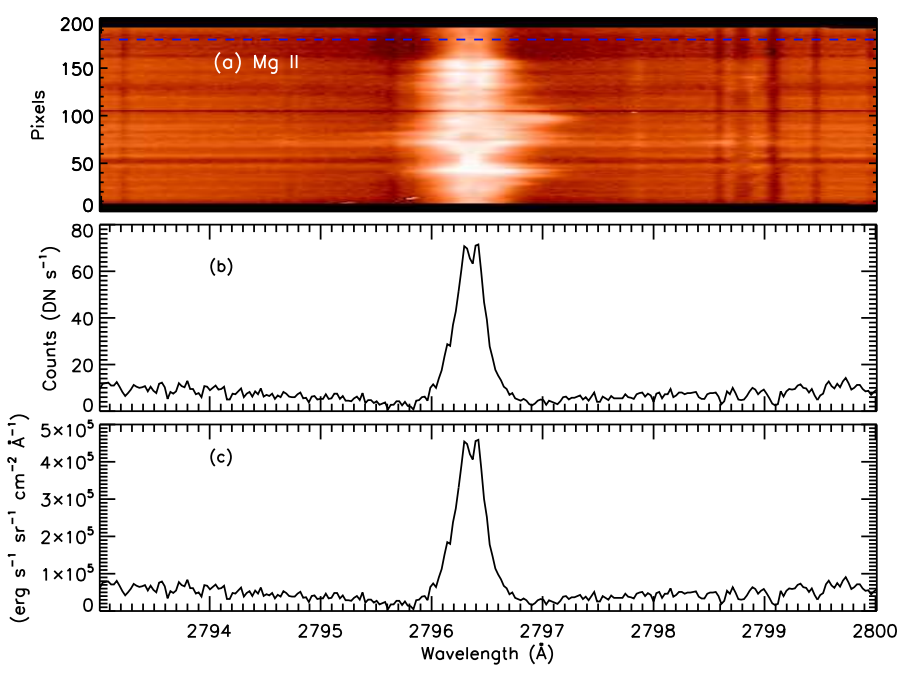

Fig. 5. Mg II k line spectra along the slit at 01:43:41 UT before the flare (a). Panels $b$ and $c$ : Mg II $\mathrm{k}$ line profiles in position $y=180$ pixel representing the quiet Sun in $\mathrm{DN} \mathrm{s}^{-1}$ and in cgs units, respectively.

the $\mathrm{k}$ line (Kleint et al. 2017). The ratio of the transmission profile between the two peaks is $10^{-2}$. The $2832 \AA$ filter reduces the intensity of $\mathrm{Mg}$ II $\mathrm{h}$ and $\mathrm{k}$ lines by a factor nearly equal to $10^{-2}$ compared to the continuum emission (see Fig. 3 in Kleint et al. 2017).

With the presence of the two peaks of $f(\lambda)$, the increase in brightening in the SJI $2832 \AA$ may be contaminated by h and $\mathrm{k}$ lines and the Fe lines present in the two wavelength domains: $h$ and $\mathrm{k}$ and near $2830 \AA$ (Kleint et al. 2017). Fortunately, we are able to eliminate this scenario because we have the Mg II spectra at the same time, which allows us to understand the contribution of the lines versus the continuum. We considered again the $\mathrm{Mg}$ II spectra obtained simultaneously in the IRIS rasters (slit 1) and in the SJIs before and during the flare time. We thus considered two wavelength ranges, one range containing the $\mathrm{Mg}$ II $\mathrm{k}$ and $\mathrm{h}$ lines, and one range around $2832 \AA$. The two light curves versus time for each wavelength range show a peak at the time of the flare (Figs. 9b and c).

During the flare at 02:04 UT, the peak maximum of the $\mathrm{DN} / \mathrm{s}$ integrated over the wavelength range 2791-2802 $\AA$ (h and $\mathrm{k}$ range) is equal to 3 and 27 (112-85) for the continuum (2821-2832 $\AA$ ) after applying the reduction factor of the filter. The excess of the Balmer continuum is about $30 \%$ (Figs. 9d, e). It is lower that what we compute directly with the spectra and the SJI, but the wavelength ranges are wider, including the two $\mathrm{Mg}$ II lines, and for the continuum domain, it is noisy due to the presence of $\mathrm{Fe}$ lines.

Furthermore, we determined the contribution of the $\mathrm{h}$ and $\mathrm{k}$ lines in the excess of the Balmer continuum with their calibrated values after applying the decrease factor due to the $2832 \AA$ filter. In Fig. 10 we present the calibrated intensity difference curves in three wavelength domains between the preflare and flare time. Two curves correspond to the continuum (Figs. 10a, c), and one curve to the $\mathrm{Mg}$ II $\mathrm{h}$ and $\mathrm{k}$ line (Fig. 10b). After multiplying by the transmission profile of the filter, we obtained the values of the intensity in the line (peak at $6 \times 10^{4} \mathrm{erg} \mathrm{s}^{-1} \mathrm{sr}^{-1} \mathrm{~cm}^{-2} \AA^{-1}$ ) and in the continuum $\left(1 \times 10^{3} \mathrm{erg} \mathrm{s}^{-1} \mathrm{sr}^{-1} \mathrm{~cm}^{-2} \AA^{-1}\right)$ in the band around $2787 \AA$ and $\left(1.5-1.75 \times 10^{5} \mathrm{erg} \mathrm{s}^{-1} \mathrm{sr}^{-1} \mathrm{~cm}^{-2} \AA^{-1}\right)$ in the band around $2832 \AA$ ). The emission of the $\mathrm{k}$ line and at $2787 \AA$ is very much reduced due to the reduction factor of the filter. 


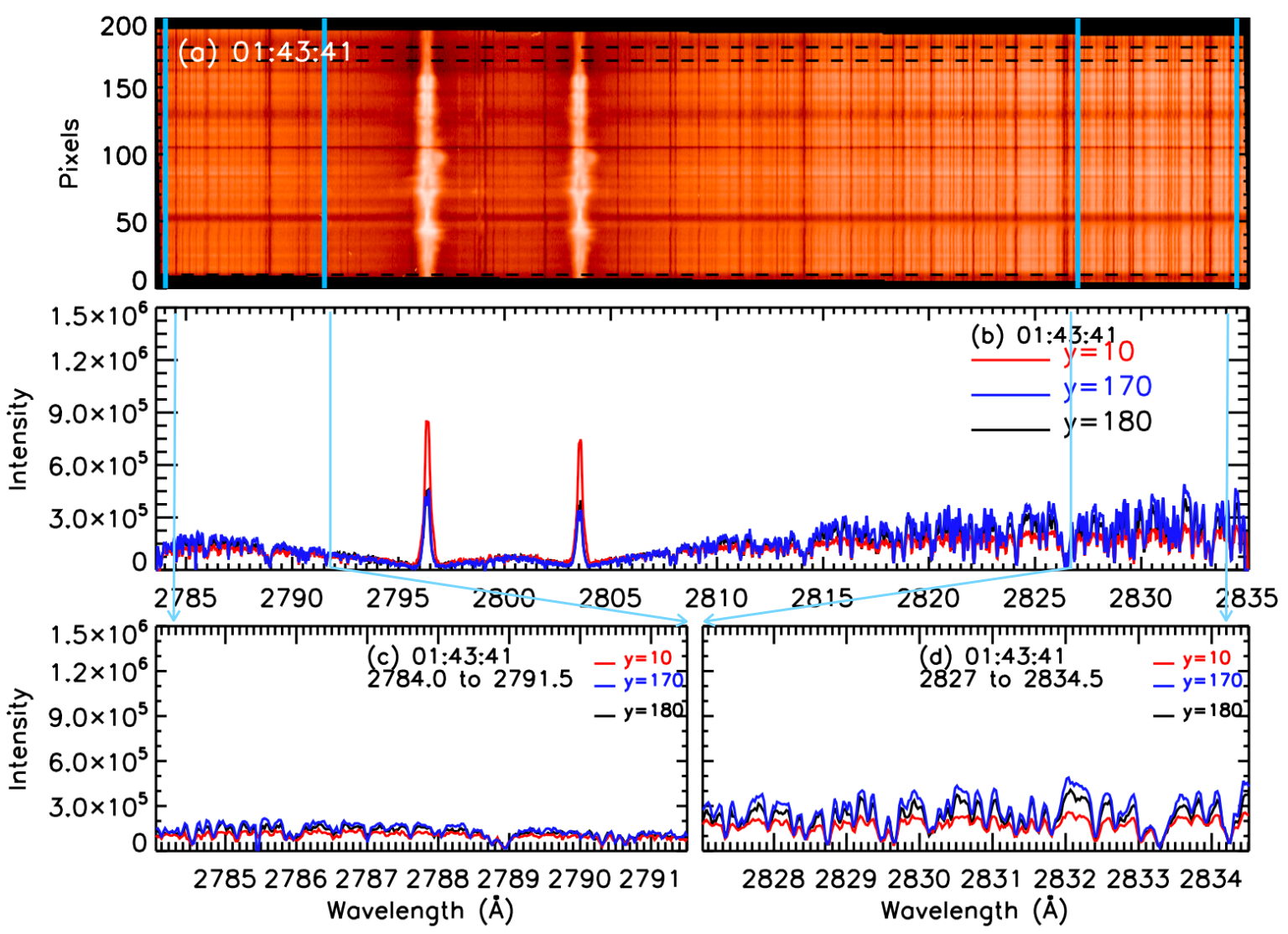

Fig. 6. Quiet-Sun intensity distribution in the IRIS Mg II wavelength band at the preflare time (01:43:41 UT). Panel a: full spectra of the Mg II band between $\lambda=2784 \AA$ and $\lambda=2835 \AA(50 \AA)$, the dashed horizontal black lines indicate $y=10,170$, and 180, which represent quiet-Sun regions. Panel b: spectral profile of the full range of $\mathrm{Mg}$ II lines for the three $y$ pixel values $(10,170$, and 180). Panels $c$ and $d$ : zoom of two parts of the spectral profile at the two ends of the wavelength band, shown by two cyan vertical lines right and left in panels a and $b$, respectively. The intensity is calibrated in $\mathrm{erg} \mathrm{s}^{-1} \mathrm{sr}^{-1} \mathrm{~cm}^{-2} \AA^{-1}$.

In a second step, we computed the integrated value of the $\mathrm{k}$ line over the wavelength range (2790-2802 $\AA$ ) and compared it to the integrated intensity of the continuum $(2820-2832 \AA$ ) (Figs. $10 \mathrm{~b}$ and c). The ratio of the total shaded areas in panel $b(20110$ for 472 pixels) and in panel c (101348 for 589 pixels) gives the contribution of the Mg II lines (around 18\%) compared to the Balmer continuum (82\%) in the wavelength domain of the 2832 filter during the flare.

In conclusion, the main contribution $(82 \%)$ of the emission in the 2832 SJIs is the Balmer continuum around $2832 \AA$ during our mini flare. This confirms the results of Kleint et al. (2017), showing that the Balmer continuum enhancement could be the principal contributor to the flare brightenings in the $2832 \AA$ SJIs in very specific pixels. Their observations concerned an X-class solar flare, in which the Balmer continuum enhancement can also be affected by the Fe II line emission. Because our flare is very weak (B class), no Fe II emission is detected. Our conclusion is therefore more convincing about the enhancement of the Balmer continuum.

\section{Fermi measurements of HXR emission}

\subsection{Fermi spectral analysis}

A significant proportion of energy released during a solar flare is thought to go into particle acceleration (Emslie et al. 2012). By studying the electron spectra obtained from the HXR photons during the flare phase, we can deduce important infor- mation about the energetics of the nonthermal electrons. For our study, we used Fermi/GBM data (12 NaI detectors), which cover the range between $8 \mathrm{keV}$ to $1 \mathrm{MeV}$ and provide 128 quasilogarithmically spaced energy bins with 4.096 s temporal resolution for several detectors with different viewing angles. We selected data from the most sunward NaI detector (detector 5).

Figure 4 shows the Fermi/GBM count rates in two energy bands for the most sunward NaI detector (detector 5) for the time period of the IRIS event analyzed in the previous section. Several HXR peaks are detected by Fermi/GBM below $14.6 \mathrm{keV}$ at the time of the GOES B-class flare, with one of these peaks detected above $14.6 \mathrm{keV}$ (time interval 02:04:43 to 02:06:34 UT). The spectral analysis performed during this time interval between 8 and $25 \mathrm{keV}$ is shown in Fig. 11. Using the OSPEX module in SSW, we fit a variable isothermal model (blue line in Fig. 11) and the nonthermal thick target component thick2, which directly provides the nonthermal electron spectrum from the fit (red line in Fig. 11). The electron spectrum (Fig. 11) was chosen as a broken power law. The best fit is obtained for the following parameters: spectral index -2.97 between $13.9 \mathrm{keV}$ and $62.7 \mathrm{keV}$, and -5.2 above $62.7 \mathrm{keV}$. The number of nonthermal electrons produced in the flare as well as the nonthermal energy contained in these nonthermal electrons can be deduced from these parameters. One of the main uncertainties on these numbers arises from the determination of the low-energy cutoff because of the dominant thermal emission at low X-ray energies.

The best fit is obtained for a low-energy cutoff of $13.9 \mathrm{keV}$, but allowing a variation of the minimum chi-square of $2 \%$ 

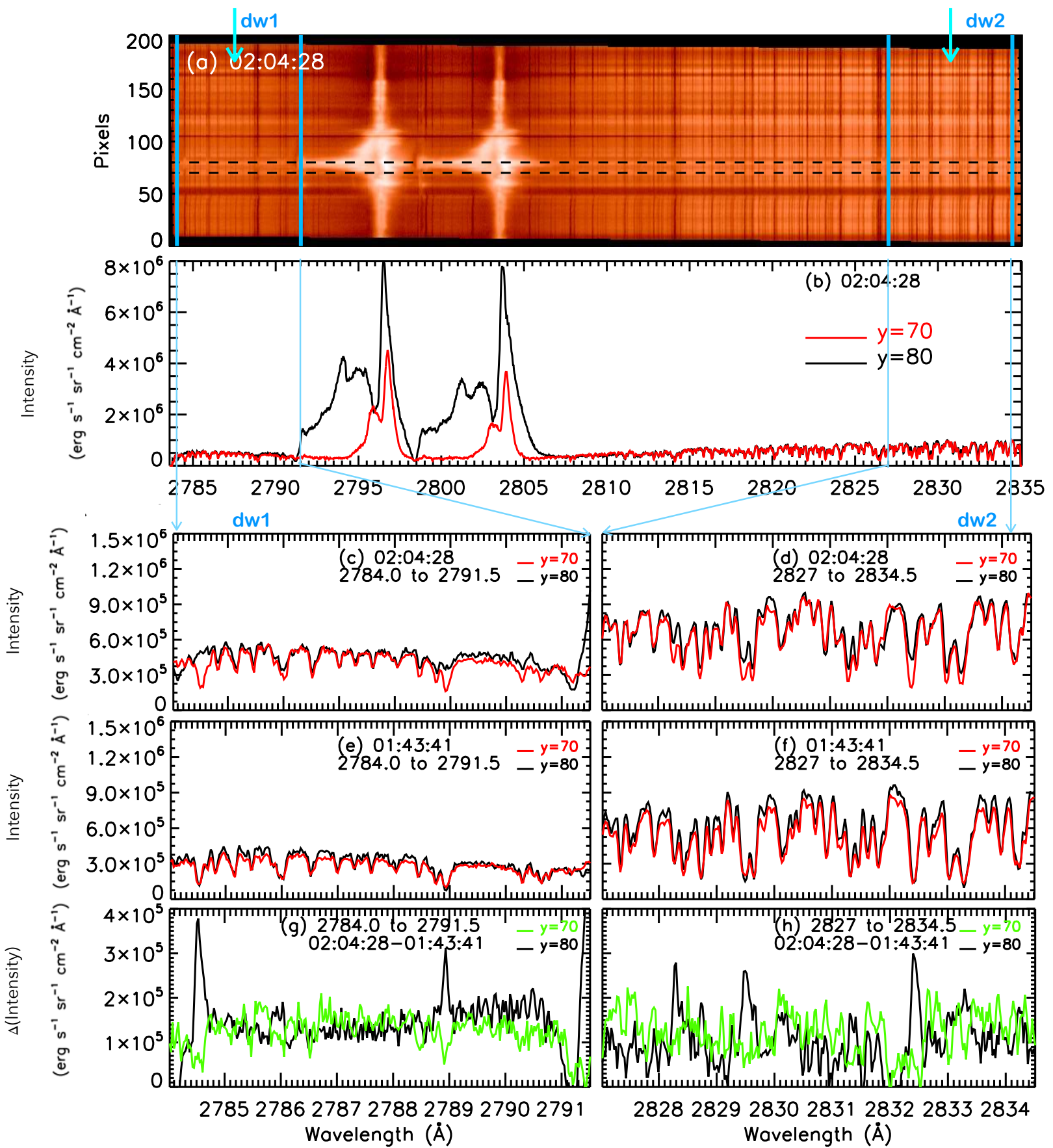

2828282928302831283228332834

Wavelength $(\AA)$

Fig. 7. Flare intensity distribution in the IRIS Mg II wavelength band at the flare time (02:04:28 UT). Panel a: full spectra of the Mg II band between $\lambda=2784 \AA$ and $\lambda=2835 \AA(50 \AA)$ at the position of slit 1 . Two dashed horizontal black lines indicate the coordinates $y=70$ and $y=80$ at the location of the maximum of the Balmer continuum in the spectral image. Panel $b$ : spectral profile in the full range of the IRIS Mg II wavelength band at the two positions ( $y=70$ and 80 pixels). Panels $c$ and $d$ : zoom of two spectral profiles of panel $\mathrm{b}$ at $(y=70,80)$ located at the two extremities of the wavelength band $(\mathrm{dw} 1, \mathrm{dw} 2)$ indicated by two vertical cyan lines and two cyan arrows at the top of panel a right and left in the panel. Panels $e$ and $f$ : similar zoom spectra in the two continuum regions at the preflare time (01:43:41). Panels $g$ and $h$ : difference in intensity $(\Delta$ (Intensity)) between the intensity at the flare time $(02: 04: 28 \mathrm{UT})$ and at the preflare time (01:43:41 UT). The intensity is calibrated to the c.g.s. units, i.e., $\operatorname{erg~s}^{-1} \mathrm{sr}^{-1} \mathrm{~cm}^{-2} \AA^{-1}$.

gives a range of possible values for the low-energy cutoff going from 10.3 to $19.5 \mathrm{keV}$. Computing the number of energetic electrons above the cutoff gives a number in the range $1.51 \times 10^{33}-$ $4.86 \times 10^{32}$. For comparison with the production of the Balmer continuum, a useful quantity is the nonthermal energy input rate per unit area. Because Fermi does not have an imaging capability, we are unable to measure the area of the HXR emitting region, but we can expect from HXR measurements with RHESSI that the HXR footpoint area is about $3^{\prime \prime}$ (e.g., Dennis \& Pernak 2009 for HXR footpoint measurements). An area of $3 \operatorname{arcsec}^{2}\left(3^{\prime \prime} \times 1^{\prime \prime}\right)$ would lead to an energy input rate of $5.4 \times 10^{8} \mathrm{erg} \mathrm{s}^{-1} \mathrm{~cm}^{-2}$. Another estimate for the area can be made using IRIS observations, as was suggested in Kleint et al. (2016), where the area was evaluated by measuring the cross 


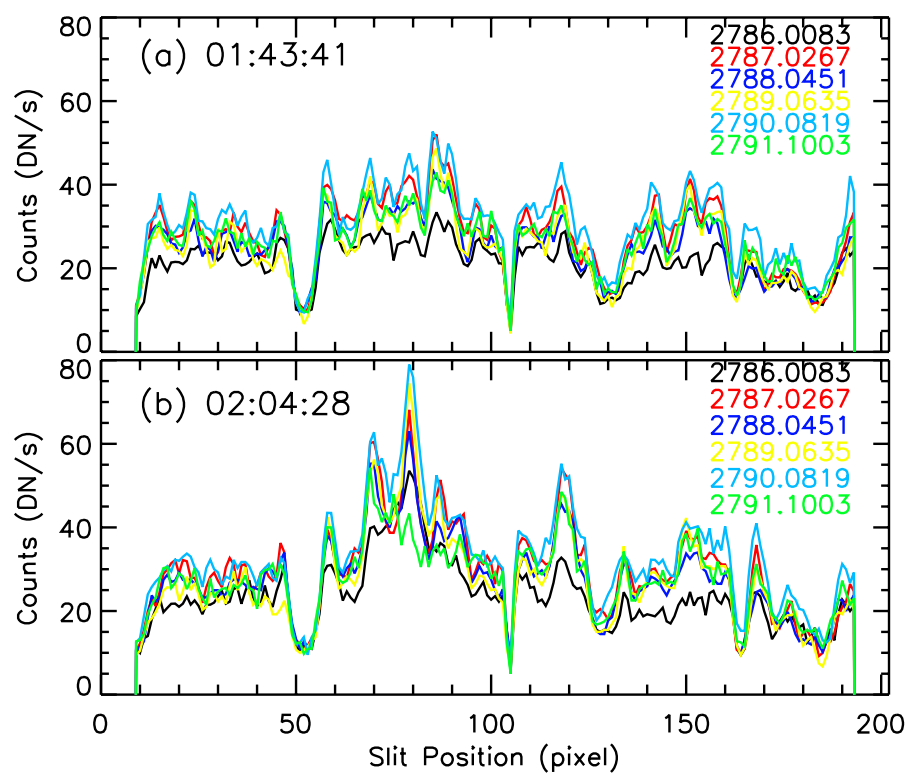

Fig. 8. Flare intensity variation along the IRIS slit at different wavelengths at the preflare (01:43:41 UT) (panel $a$ ) and flare (02:04:28 UT) (panel $b$ ) times. The different colors of the curves show the different wavelengths. The intensity variation is constant at the preflare time, whereas the curve shows two peaks at $y=70$ and 80 pixel at the flare time. The counts are lowest at the slit position $y=180$ pixel. These three positions are chosen as the reference of the quiet-Sun and the Balmer enhancement.

section of the ribbon along the IRIS slit. The spatial resolution of IRIS is $0.33^{\prime \prime}$. Thus if we assume an upper limit of $0.5 \times 0.5 \operatorname{arcsec}$ for the area of the deposition of nonthermal energy, the energy input rate per unit area produced by electrons above $20 \mathrm{keV}$ is $6.5 \times 10^{9} \mathrm{erg} \mathrm{s}^{-1} \mathrm{~cm}^{-2}$.

\subsection{Non-LTE radiative transfer models}

The second step of our study was to estimate whether the energy needed for the Balmer continuum excess might correspond to the energy brought by the bombardment of nonthermal electrons. We need to compare the energy input provided by nonthermal electrons during the mini flare with the excess of the Balmer continuum enhancement observed by IRIS. This type of comparison was done previously by Kleint et al. (2016), who presented IRIS and RHESSI data for a strong X-ray flare. To do this, they considered a theoretical grid of 1D static flare atmosphere models developed by Ricchiazzi \& Canfield (1983), usually referred to as the RC models, in which the temperature structure is computed through the energy balance between the electron-beam heating, conduction, and net radiation losses and using as a free parameter the enhancement of the coronal pressure due to evaporation. Furthermore, Kleint et al. (2016) used the non-LTE code MALI to synthesize the hydrogen recombination continua for all the models. They compared the resulting intensities of the Balmer continuum to those detected by IRIS. The RC flare models thus provide a relation between the electron-beam energy flux with the cutoff energy $20 \mathrm{keV}$ having a given spectral index and the enhancement of the Balmer continuum. Our observed excess of the Balmer continuum intensity is about 1.5 to $1.75 \times 10^{5} \mathrm{erg} \mathrm{s}^{-1} \mathrm{sr}^{-1} \mathrm{~cm}^{-2} \AA^{-1}$ (Figs. $7 \mathrm{~g}$ and h, and Fig. 10c). According to the results of Kleint et al. (2016) (see their Table 1), this value is roughly consistent with a beam flux of $10^{9}$ and $10^{10} \mathrm{erg} \mathrm{s}^{-1} \mathrm{~cm}^{-2}$ (RC models E5

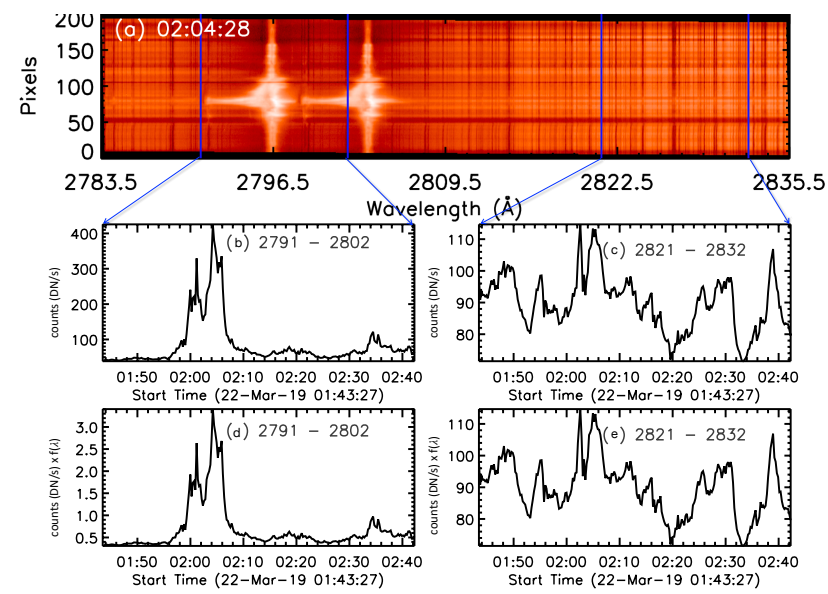

Fig. 9. Evolution vs. time of the intensity in the Mg II k and in the $2832 \AA$ ranges. Top panel: IRIS spectra of the Mg II band along slit position 1. The two wavelength regions in $\mathrm{Mg}$ II k line (left) and in the Balmer emission (right) are bounded by vertical blue lines. Bottom panels: temporal evolution of the relative contribution of $\mathrm{Mg}$ II $\mathrm{k}$ line $(b, d)$ and Balmer continuum emission $(c, e)$ at the reconnection point of the flare $(y=80)$. Panels $c$ and $d$ : the intensity (DN) has been divided by the transmission factor $\mathrm{f}(\lambda)$ of the 2832 filter taken from Kleint et al. (2017). The comparison of the relative peak values at the flare time in $\mathrm{Mg}$ II range (panel c) and continuum region (panel $d$ ) indicates the prevalence of the Balmer continuum.

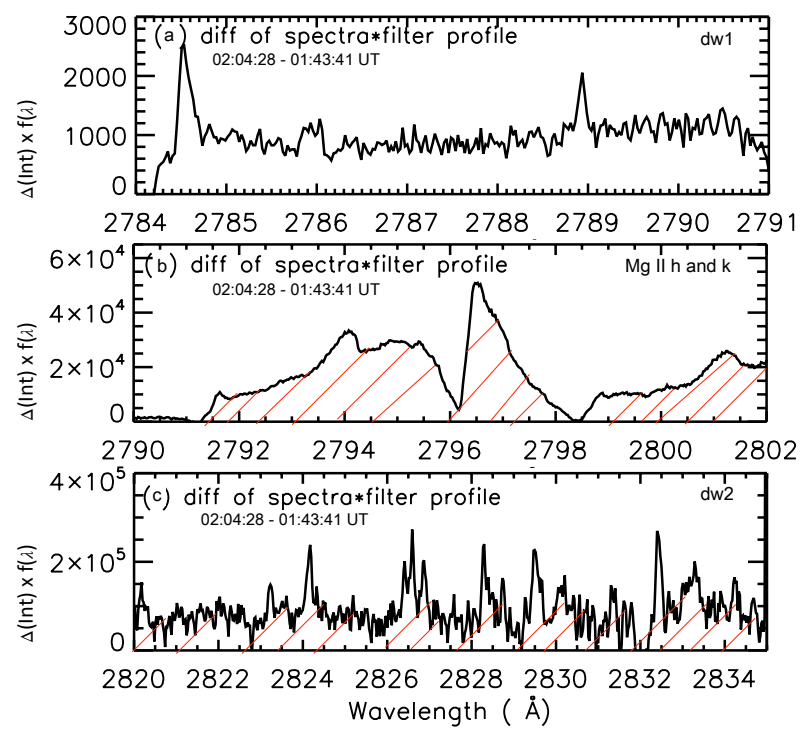

Fig. 10. Variation with wavelength of the difference of intensity between flare and preflare times in three wavelength ranges containing continuum regions (dw1 and dw2) and the $\mathrm{Mg}$ II $\mathrm{h} \& \mathrm{k}$ line. The differences in intensity have been multiplied by the transmission filter profile $\mathrm{f}(\lambda)$ of the 2832 A filter (Kleint et al. 2017). After normalization of the hatched areas in panels $b$ and $c$, we estimate that the contribution of the excess of the Balmer emission is $82 \%$ compared to the contribution of the $\mathrm{Mg}$ II $\mathrm{k}$ line around $18 \%$ in the wavelength domain of the $2832 \AA$ SJI during the flare.

and E12). Such fluxes have been derived from Fermi/GBM observations, as we show above. For model E5, the coronal pressure is $100 \mathrm{dyn} \mathrm{cm}^{-2}$, which leads to very bright $\mathrm{Mg}$ II line cores as computed in Liu et al. (2015), which are not observed in our weak flare (such a high pressure is found in an X-class flare atmosphere analyzed by Liu et al. 2015). The second model E12 with the lower coronal pressure predicts Mg II line-core 

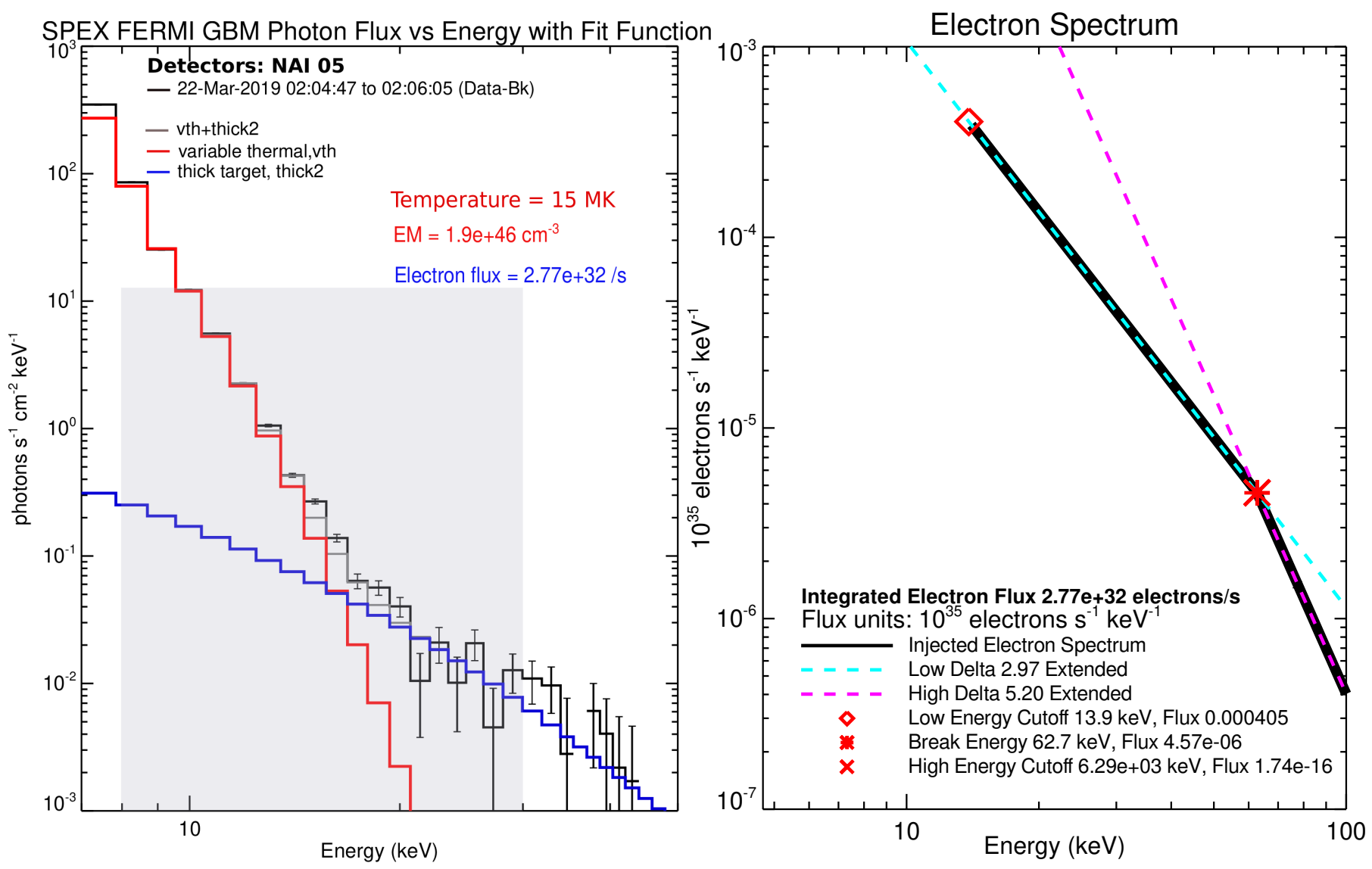

Fig. 11. Fermi/GBM photon spectra accumulated around 02:04:47 UT and 02:06:05 UT during the quoted 2-min interval on March 22, 2019 (left panel). Variable isothermal and thick target fitting was performed on the Fermi/GBM spectrum. For the variable thermal, we used the ' $v$ th' function, and for thick target, we used 'thick2' from OSPEX. The variable thermal fit is shown by the red curve, and the thick target fit is shown by the blue curve. The fit is limited to between 8 and $30 \mathrm{keV}$ shown by the shaded region. Above $30 \mathrm{keV}$, the counts are predominantly that of background. The fit shown here is the best fit obtained using $\chi^{2}$ as a measure. We find that a range of values for low-energy cutoff parameter $(10.3-19.5 \mathrm{keV})$ gives reasonable fit. The electron spectrum given by the thick-target fit is shown in the right panel.

intensities that are more compatible with our IRIS observations and is thus reasonable for this weak flare. The energy value is on same order as the energy brought by the electron beams of Fermi/GBM between $10^{9}$ and $10^{10} \mathrm{erg} \mathrm{s}^{-1} \mathrm{~cm}^{-2}$ due to the uncertainty of the area size.

\section{Discussion and conclusion}

We extended the analysis of the IRIS data of GOES B6.7 micro flare (or mini flare) observed on March 22, 2019, focusing on the IRIS SJIs in the $2832 \AA$ filter and the NUV continuum spectral variation in the two extreme wavelength domains of the IRIS $2896 \AA$ spectra bandpass (between 2784-2791.5 $\AA$ and 2827-2834.5 $\AA$ ). The mini flare and its site of reconnection identified by bidirectional outflows concern only one or two pixels along slit position 1 of the IRIS rasters (Joshi et al. 2021). Therefore we focused our analysis on the spectra along this slit. Throughout the paper, we demonstrated that the enhancement of the NUV continuum was due to electron beams and not to the flare heating by interplaying the analysis of IRIS images and spectra.

A brightening in the 2832 filter is visible in the area of the flare. After considering the wavelength domain of the filter, its transmission function, and the emission of the spectra in the same spectral domain, we conclude that this was the direct signature of electron beams. The excess of the Balmer continuum in the spectra detected at the flare time in the few considered pixels is constant throughout the two considered spectral ranges (bottom panels of Fig. 7). The increment in the continuum intensity being constant cannot be due to the intensity enhancement in the far wings of $\mathrm{Mg}$ II lines, which decreases with distance from the k $(2796 \AA)$ or h $(2803 \AA) \mathrm{Mg}$ II line center on either side. The Balmer continuum being optically thin in a weak flare, thus its emission really far from $\mathrm{Mg}$ II h (more than $30 \AA$ ) for the second range is simply added to the photospheric (pre-flare) background as a constant excess of intensity over the photospheric continuum.

The cotemporal time of the continuum enhancement peak in the spectral profiles and in SJIs suggests that the SJI brigthness is dominated by the Balmer enhancement continuum. We found that the contribution of the Balmer continuum is about $82 \%$ compared to the $18 \%$ of the extended $\mathrm{Mg}$ II line wings in the spectral domain of the 2832 filter. In addition, the NUV continuum in this wavelength range is relatively simple to analyze because the metallic lines (mainly Fe II lines) present in this domain are not in emission for this mini flare. This is a very different case from previous studies concerning X-class flares where metallic lines in the spectra around $2830 \AA$ went into emission and affected the Balmer continuum (Heinzel \& Kleint 2014; Kleint et al. 2017). Previous authors had to distinguish between two emissions: continuum, and Fe II lines.

For our weak flare, we demonstrated that the Fermi/GBM energy output by nonthermal electrons is consistent with the 
beam flux required in non-LTE radiative models to obtain the Balmer continuum emission excess measured in the IRIS spectra. In large flares, it has been shown that the injected nonthermal energy derived from RHESSI data is sufficient and even in excess to explain the thermal component of strong flares (Aschwanden et al. 2017; Kleint et al. 2016). However, it was shown that for weak flares, a deficit of energetic electrons to affect the low levels of the atmosphere in which the bolometric emission (NUV, white light, near-IR radiation) is initiated (Warmuth \& Mann 2020). Inglis \& Christe (2014), Warmuth \& Mann (2020) reported an apparent deficit of nonthermal electrons in weak flares by computing the ratio of thermal energy (and losses) and energy in nonthermal electrons. The interpretation depends on where and in which area the electrons are accelerated. In the strong energetic flares studied by Heinzel \& Kleint (2014) and Kleint et al. (2017), the electron beam energy was high enough to power the thermal flare, as the Ricchiazzi \& Canfield (1983) models demonstrated.

In our case, the reconnection site of the mini flare at the base of the jet was identified to lie in a tiny bald-patch region transformed dynamically in a X-point current sheet, which explains its multithermal components (Joshi et al. 2020). The electron beam input should be sufficient to power the thermal flare observed with Balmer continuum excess. The estimation of the nonthermal energy is based on the size of the deposit electron area. This is a relatively unknown variable. The site of reconnection may be smaller than the IRIS spatial resolution, and the energy input per unit area is maybe underestimated. The spectral signatures of the mini flare have been identified as IRIS bomb spectra (Peter et al. 2014; Grubecka et al. 2016; Young et al. 2018; Joshi et al. 2021). These structures can be due to plasmoid instablity, which creates tiny multi-thermal plasmoids that are not resolved by our telescopes (Ni et al. 2016, 2021; Baty 2019).

This study shows that the nonthermal HXR emission detected by Fermi/GBM is strongly related to the enhancement of the Balmer continuum emission, which is a signature of a significant excess in heating. We therefore conclude that the detection of the Balmer continuum emission in our mini flare supports the scenario of hydrogen recombination in flares after a sudden ionization at chromospheric layers.
Acknowledgements. We thank the referee for his/her numerous insightful comments which have greatly helped to improve the manuscript. IRIS is a NASA small explorer mission developed and operated by LMSAL with mission operations executed at NASA Ames Research center and major contributions to downlink communications funded by ESA and the Norwegian Space Centre. We thank Jana Kasparova for the discussion and suggestions. We are thankful to Hui Tian and Krzysztof Barczynski for the discussion regarding the IRIS data calibration. RJ thanks to CEFIPRA for a Raman Charpak Fellowship (RCF-IN00136) under which this work is initiated at the Observatoire de Paris, Meudon. RJ also acknowledges the support from Department of Science and Technology (DST), New Delhi, India as an INSPIRE fellow. PH acknowledges support from the Czech Funding Agency, grant 19-09489S. RC acknowledges the support from Bulgarian Science Fund under Indo-Bulgarian bilateral project, DST/INT/BLR/P-11/2019.

\section{References}

Aschwanden, M. J., Caspi, A., Cohen, C. M. S., et al. 2017, ApJ, 836, 17 Baty, H. 2019, ApJS, 243, 23

Dennis, B. R., \& Pernak, R. L. 2009, ApJ, 698, 2131

De Pontieu, B., Title, A. M., Lemen, J. R., et al. 2014, Sol. Phys., 289, 2733

Ding, M. D., Liu, Y., Yeh, C. T., \& Li, J. P. 2003, A\&A, 403, 1151

Emslie, A. G., Dennis, B. R., Shih, A. Y., et al. 2012, ApJ, 759, 71

Fletcher, L., Dennis, B. R., Hudson, H. S., et al. 2011, Space Sci. Rev., 159, 19

Grubecka, M., Schmieder, B., Berlicki, A., et al. 2016, A\&A, 593, A32

Heinzel, P., \& Kleint, L. 2014, ApJ, 794, L23

Inglis, A. R., \& Christe, S. 2014, ApJ, 789, 116

Joshi, R., Schmieder, B., Aulanier, G., Bommier, V., \& Chandra, R. 2020, A\&A, 642, A169

Joshi, R., Schmieder, B., Tei, A., et al. 2021, A\&A, 645, A80

Kleint, L., Heinzel, P., Judge, P., \& Krucker, S. 2016, ApJ, 816, 88

Kleint, L., Heinzel, P., \& Krucker, S. 2017, ApJ, 837, 160

Lemen, J. R., Title, A. M., Akin, D. J., et al. 2012, Sol. Phys., 275, 17

Liu, W., Heinzel, P., Kleint, L., \& Kašparová, J. 2015, Sol. Phys., 290, 3525

Machado, M. E., Emslie, A. G., \& Avrett, E. H. 1989, Sol. Phys., 124, 303

Meegan, C., Lichti, G., Bhat, P. N., et al. 2009, ApJ, 702, 791

Neidig, D. F. 1989, Sol. Phys., 121, 261

Ni, L., Lin, J., Roussev, I. I., \& Schmieder, B. 2016, ApJ, 832, 195

Ni, L., Chen, Y., Peter, H., Tian, H., \& Lin, J. 2021, A\&A, 646, A88

Pereira, T. M. D., Leenaarts, J., De Pontieu, B., Carlsson, M., \& Uitenbroek, H. 2013, ApJ, 778, 143

Pesnell, W. D., Thompson, B. J., \& Chamberlin, P. C. 2012, Sol. Phys., 275, 3

Peter, H., Tian, H., Curdt, W., et al. 2014, Science, 346, 1255726

Ricchiazzi, P. J., \& Canfield, R. C. 1983, ApJ, 272, 739

Simões, P. J. A., Reid, H. A. S., Milligan, R. O., \& Fletcher, L. 2019, ApJ, 870, 114

Warmuth, A., \& Mann, G. 2020, A\&A, 644, A172

Young, P. R., Tian, H., Peter, H., et al. 2018, Space Sci. Rev., 214, 120 